Revue d'histoire de l'Amérique française

REVUE D.HISTOIRE DE L'AMÉRIQUE FRANÇAISE

\title{
Les conceptions prénuptiales dans la vallée du Saint-Laurent avant 1725
}

\section{Réal Bates}

Volume 40, numéro 2, automne 1986

URI : https://id.erudit.org/iderudit/304447ar

DOI : https://doi.org/10.7202/304447ar

Aller au sommaire du numéro

Éditeur(s)

Institut d'histoire de l'Amérique française

ISSN

0035-2357 (imprimé)

1492-1383 (numérique)

Découvrir la revue

Citer cette note

Bates, R. (1986). Les conceptions prénuptiales dans la vallée du Saint-Laurent avant 1725. Revue d'histoire de l'Amérique française, 40(2), 253-272.

https://doi.org/10.7202/304447ar
Résumé de l'article

La statistique démographique permet de mesurer les naissances illégitimes et les conceptions prénuptiales pour les populations anciennes, offrant ainsi un éclairage nouveau sur leurs moeurs. Les deux contributions qui suivent offrent des résultats inédits concernant ces deux phénomènes dans la vallée du Saint-Laurent d'avant 1730. Si les interdits religieux en matière de sexualité hors mariage ne furent pas toujours respectés, il n'en demeure pas moins que seulement 1,25\% de l'ensemble des naissances sont illégitimes et $6,1 \%$ des premières naissances ont été conçues avant le mariage. Une évolution se dessine cependant dans le temps, de même que des distinctions doivent être faites selon le milieu urbain ou rural. L'état matrimonial des mères ainsi que leur âge sont aussi des facteurs déterminants de la fréquence de ces comportements. Signalons enfin que le mouvement saisonnier de ces conceptions hors mariage, sensiblement différent de celui des conceptions légitimes, et la très forte surmortalité des bâtards ne manquent pas d'intérêt pour l'étude de cette population ancienne.
Tous droits réservés @ Institut d'histoire de l'Amérique française, 1986
Ce document est protégé par la loi sur le droit d'auteur. L’utilisation des services d'Érudit (y compris la reproduction) est assujettie à sa politique d'utilisation que vous pouvez consulter en ligne.

https://apropos.erudit.org/fr/usagers/politique-dutilisation/ 


\title{
LES CONCEPTIONS PRÉNUPTIALES DANS LA VALLÉE DU SAINT-LAURENT AVANT 1725 ${ }^{1}$
}

\author{
RÉAL BATES \\ Programme de recherche en démographie historique \\ Université de Montréal
}

«L'oeuvre de chair ne désireras qu'en mariage seulement». (9e commandement de Dieu)

\section{INTRODUCTION}

La vie sexuelle des populations anciennes est devenue depuis quelques années un des thèmes féconds de l'historiographie moderne: les oeuvres des historiens Flandrin, Shorter et Laslett représentent bien tout l'intérêt de telles recherches. Ce thème d'étude - si éclairant sur les moeurs des populations françaises et anglaises d'Ancien Régime n'a pas encore son équivalent au Canada français. Certes les historiens de la Nouvelle-France n'ont pas manqué de dresser un portrait moral des premiers Canadiens. L'historiographie traditionnelle, inspirée de l'idéologie cléricale du $19 \mathrm{e}$ siècle et de la première moitié du $20 \mathrm{e}$ siècle ${ }^{2}$, s'est surtout complue à dresser un portrait idyllique du comportement moral des anciens Canadiens ou à défendre la vertu de nos ancêtres féminines dont la réputation semblait attaquée ${ }^{3}$.

Pour prendre le contre-pied de cette optique traditionnelle, RobertLionel Séguin tenta de montrer que «galanterie, libertinage et marivaudage ont leur place en la nouvelle comme en l'ancienne France» ${ }^{4}$. Adultère, bigamie, viol, séduction, prostitution, etc., existaient au $17 \mathrm{e}$ siècle, et nos ancêtres auraient alors été «des êtres normaux» ${ }^{5}$.

1 Ce texte présente les principales observations faites dans le cadre de notre mémoire de maîtrise, Les conceptions prénuptiales dans la vallée du Saint-Laurent avant 1725, Université de Montréal (démographie), 1985, 178 p. Nous tenons à remercier ici notre directeur de recherche Hubert Charbonneau ainsi que le Conseil de recherches en sciences humaines du Canada qui a permis la réalisation de cet article.

Jean Blain, "La moralité en Nouvelle-France: les phases de la thèse et de l'antithèse», Revue d' histoire de l'Amérique française, 27,3 (décembre 1973): 408-416.

3 Gustave Lanctôt, Filles de joie ou Filles du Roi. Étude sur l'émigration féminine en Nouvelle-France (Montréal, Les éditions Chanteclerc Ltée, 1952), 230 p.

4 Robert-Lionel Séguin, La vie libertine en Nouvelle-France au dix-septième siècle (Montréal, Leméac, 1972), 546.

5 Ibid.

RHAF, vol. 40, no 2, automne 1986 
Avouons que le concept de «normalité» utilisé par Séguin n'apprend pas grand-chose. Nul ne doute que toute société connaît des amours illégitimes, des viols, des adultères, etc. L'important serait de connaître non seulement la nature, mais aussi l'intensité de ces phénomènes, ainsi que l'acceptation ou la réprobation sociale à laquelle ils étaient soumis. Les faits recensés par Séguin, dans la mesure où ils sont surtout connus par les archives judiciaires, nous renseignent autant, sinon plus, sur la réprobation sociale dont ils étaient l'objet que sur les faits eux-mêmes.

Dans l'état actuel des recherches, il faut bien convenir que nous savons peu de chose des moeurs de nos ancêtres. Plutôt que de vouloir juger de la «moralité» de ceux-ci, démarche stérile, en se basant sur des témoignages qualitatifs, peut-être conviendrait-il mieux d'étudier cette société. La démographie peut ici apporter sa contribution, tout au moins en ce qui concerne les phénomènes que la statistique peut saisir, et que les démographes-historiens n'ont pas manqué d'observer: naissances illégitimes et conceptions prénuptiales.

\section{1 - La morale de l'époque}

On sait jusqu'à quel degré d'austérité peut aller la morale catholique en matière sexuelle. Au sixième commandement déclarant «luxu-

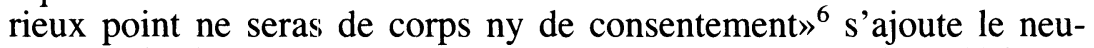
vième, plus important pour notre propos: «l'oeuvre de chair ne désireras qu'en mariage seulement» ${ }^{7}$. L'Église canadienne marquée par la réforme catholique des $16 \mathrm{e}$ (Concile de Trente) et $17 \mathrm{e}$ siècles ne dérogera certes pas à cette morale rigoureuse.

Mgr de Saint-Vallier déclare: «C'est la volonté de Dieu que nous soyons saints et purs et qu'un chacun sçache posséder son corps avec sainteté et honnêteté, et non point dans une passion déréglée, comme les Payens qui ne connaissent point Dieu.» ${ }^{8}$

Le même prélat considère même «l'impureté (...) comme une des principales causes des châtiments que Dieu nous envoye» ${ }^{9}$. Et, pour cet ecclésiastique, bien des comportements qui seraient pour nous anodins, représentent des atteintes à cette chasteté exigée. Non seulement, les robes dévoilant un peu trop le sein féminin sont-elles condamnées, mais plus encore: «Que faut-il éviter pour conserver la chasteté? Les lieux, les entretiens et les fréquentations trop libres de personnes d'un autre

6 Jean de la Croix de Saint-Vallier, Rituel du diocèse de Québec (Paris, Simon Langlois, 1703), 333.

I bid.

8 Jean de la Croix de Saint-Vallier, Catéchisme du diocèse de Québec (Montréal, Réimpression par les Éditions Franciscaines, 1958), 187.

9 Ordonnance du 31 octobre 1680 in Jean de la Croix de Saint-Vallier, Statuts, ordonnances et lettres pastorales de Monseigneur de Saint-Vallier, évêque de Québec, pour règlement de son diocèse (Paris, Simon Langlois, 1703), 13. 
sexe, la lecture des mauvais livres, les danses et les excez de bouche.» ${ }^{10}$ Même le mariage ne doit pas se réduire à l'oeuvre de chair: «Il faut convenir que ceux qui se marient par sensualité ne recherchant que les plaisirs de leurs sens dans le mariage, ou par avarice, ne regardant que l'établissement de leur fortune temporelle, commettent un très grand péché, parce qu'elles profanent le Sacrement de Mariage.» ${ }^{11}$

On peut penser que cette norme morale était partagée par l'ensemble des Français et des Canadiens de la colonie, tous catholiques, mais dans quelle mesure? L'observation des naissances illégitimes et des conceptions prénuptiales permet de constater l'observance, au sein de la population, du neuvième commandement. Les naissances illégitimes dans la Nouvelle-France d'avant 1730 ont déjà fait l'objet d'une étude ${ }^{12}$. Le présent texte présente des données concernant le phénomène des conceptions prénuptiales dans la vallée du Saint-Laurent d'avant 1725. Notons que cette étude ne dressera pas un portrait d'ensemble des moeurs sexuelles de nos ancêtres. Elle veut simplement fixer quelques faits statistiques sur un phénomène restreint: les rapports sexuels d'avant mariage ayant donné lieu à une naissance dans le cadre d'une union légitime.

\section{2 - Mode de calcul des conceptions prénuptiales}

Nos ancêtres ne nous ayant pas, bien entendu, laissé de confidences sur le moment de la conception de leur premier-né, il va de soi que c'est par déduction statistique que nous pouvons déterminer la fréquence des conceptions prénuptiales. La méthode devenue classique en démographie pour une telle estimation consiste à rapporter à l'ensemble des premières naissances toutes celles intervenues dans les huit premiers mois du mariage (mois révolus 0 à 7). Ce calcul repose sur le postulat que les possibles conceptions d'avant mariage ainsi perdues (une partie des naissances du neuvième mois, non prises en compte) compensent pour les premiers-nés conçus après le mariage et nés avant le neuvième mois (prématurés ou enfants à la gestation plus courte que la moyenne). «Ce procédé ne peut être qu'approximatif» ${ }^{13}$, mais il est celui généralement utilisé et qui permet donc les comparaisons entre populations.

\section{3 - Sources}

C'est grâce au travail des artisans du Programme de recherche en démographie historique (PRDH) que notre recherche a pu être menée.

\footnotetext{
10 Saint-Vallier, Catéchisme..., 188.

11 Saint-Vallier, Rituel..., 280.

12 Voir la note de recherche précédente et Lyne Paquette, Les naissances illégitimes sur les rives du Saint-Laurent avant 1730. Mémoire de maîtrise, Université de Montréal (démographie), 1983, $202 \mathrm{p}$.

${ }_{13}$ Louis Henry, Techniques d'analyse en démographie historique (Paris, Institut national d'études démographiques, 1980), 177 p.
} 
Nous avons pu en effet disposer d'un fichier informatisé contenant toutes les informations nécessaires pour toutes les unions formées avant 1725 dans la vallée du Saint-Laurent et pour lesquelles la reconstitution automatique des familles est chose faite. C'est donc dire que notre étude ne se base pas sur un échantillon, comme ce fut le cas pour les études, aujourd'hui devenues classiques, de Henripin ${ }^{14}$ et Charbonneau ${ }^{15}$.

Il nous a fallu cependant faire une certaine sélection parmi les 9 831 unions concernées. Dans un premier temps, les 1136 unions restées infécondes ${ }^{16}$ ont été rejetées, pour des raisons évidentes. Quant aux autres, nous n'avons retenu que les unions pour lesquelles et la date de mariage et la date de naissance du premier enfant étaient exactes (dates extraites d'un acte de mariage et d'un acte de baptême) et complètes. Sept mille cent quatre-vingt-trois unions (soit $83 \%$ des unions fécondes formées entre 1621 et 1725) ont donc servi à cette étude. Le rejet est relativement peu important, ce qui prouve la qualité de la banque de données du PRDH, et peut difficilement causer de biais puisque les unions rejetées le sont à cause de lacunes dans les registres paroissiaux conservés à ce jour, lacunes ne pouvant avoir de lien avec l'existence du phénomène étudié ${ }^{17}$.

\section{4 - L'ampleur du phénomène}

Plus élevée dans le premier quart du $18 \mathrm{e}$ siècle qu'au $17 \mathrm{e}$ siècle (voir tableau 1), la fréquence des conceptions prénuptiales est de l'ordre

TABLEAU 1

Conceptions prénuptiales au Canada avant 1725

\begin{tabular}{lccc}
\hline & \multicolumn{3}{c}{ Périodes } \\
\hline & $1621-1699$ & $1700-1724$ & $1621-1724$ \\
\hline $\begin{array}{l}\text { Nombre de premières naissances } \\
\text { Intervalles protogénésiques } \\
\text { inférieurs à huit mois révolus }\end{array}$ & 3054 & 4129 & 7183 \\
$\begin{array}{l}\text { Proportion de conceptions } \\
\text { prénuptiales (\%) }\end{array}$ & 140 & 296 & 436 \\
\hline
\end{tabular}

14 Jacques Henripin, La population canadienne au début du XVIIIe siècle (Paris, Presses universitaires de France, 1954), 129 p.

15 Hubert Charbonneau, Vie et mort de nos ancêtres (Montréal, Presses de l'Université de Montréal, 1975), $267 \mathrm{p}$.

${ }_{16}$ Infécondes au moins jusqu'au 31 décembre 1729, fin de la période d'observation pour l'actuelle reconstitution des familles au PRDH. C'est donc dire que certaines de ces unions ont pu avoir un premier enfant après cette date et qu'en conséquence notre dénominateur serait sousestimé. Mais cette sous-estimation ne peut être que légère, les intervalles protogénésiques très longs (plus de 5 ans pour les unions formées en 1724, 6 ans pour celles de 1723, etc.) ne pouvant être que très rares dans une population à fécondité non dirigée. Notons également que pour le calcul de la fréquence des conceptions prénuptiales nous avons utilisé les 7183 unions sans tenir compte de leur durée.

17 Pour plus de précision sur la question méthodologique et l'estimation des biais éventuels de notre sélection, voir notre mémoire, op. cit., 3-41. 
TABLEAU 2

Conceptions prénuptiales en Nouvelle-France selon divers auteurs

\begin{tabular}{|c|c|c|c|c|c|c|}
\hline Lieu & Époque & $\begin{array}{c}\text { Nombre de } \\
\text { premières } \\
\text { naissances }\end{array}$ & $\begin{array}{l}\text { Nombre de } \\
\text { conceptions } \\
\text { prénuptiales }\end{array}$ & $\begin{array}{l}\text { Caractéristiques } \\
\text { des populations }\end{array}$ & $\begin{array}{c}\text { Pourcentage } \\
\text { de } \\
\text { conceptions } \\
\text { prénuptiales }\end{array}$ & Source \\
\hline Canada & $\begin{array}{l}17 \mathrm{e} \\
\text { siècle }\end{array}$ & 854 & 33 & $\begin{array}{l}\text { femmes de moins } \\
\text { de vingt ans }\end{array}$ & 3,9 & (1) \\
\hline Canada & $\begin{array}{l}17 \mathrm{e} \\
\text { siècle }\end{array}$ & 2828 & 130 & & 4,6 & (2) \\
\hline Canada & $\begin{array}{l}17 \mathrm{e} \\
\text { siècle }\end{array}$ & 362 & 16 & & 4,5 & (3) \\
\hline Canada & $1700-1759$ & $\begin{array}{l}542 \\
352\end{array}$ & $\begin{array}{l}46 \\
25\end{array}$ & $\begin{array}{l}\text { premier mariage } \\
\text { deuxième mariage }\end{array}$ & $\begin{array}{l}8,5 \\
7,1\end{array}$ & $\begin{array}{l}(4) \\
\text { (4) }\end{array}$ \\
\hline Canada & $\begin{array}{l}17 \mathrm{e} \\
\text { siècle }\end{array}$ & & & $\begin{array}{l}\text { villes } \\
\text { campagnes }\end{array}$ & $\begin{array}{l}4,8 \\
3,4\end{array}$ & $\begin{array}{l}(5) \\
(5)\end{array}$ \\
\hline Canada & $1700-1730$ & 725 & 59 & & 8,1 & (6) \\
\hline Canada & $\begin{array}{l}17 \mathrm{e} \\
\text { siècle }\end{array}$ & 407 & 19 & & 4,7 & (7) \\
\hline Beauport & $1634-1762$ & 381 & 27 & & 7,1 & $(8)$ \\
\hline $\begin{array}{l}\text { Canada } \\
\text { (noblesse) }\end{array}$ & $1687-1779$ & 32 & 2 & $\begin{array}{l}\text { famille noble: } \\
\text { les D'Ailleboust }\end{array}$ & 6,25 & (9) \\
\hline Neuville & $1669-1762$ & 321 & 20 & & 6,2 & $(10)$ \\
\hline $\begin{array}{l}\text { Cap- } \\
\text { Santé, } \\
\text { Descham- } \\
\text { bault, } \\
\text { Grondines }\end{array}$ & 1679-1762 & 302 & 26 & & 8,6 & (11) \\
\hline
\end{tabular}

Sources:

(1) Carmen Bellerose, La fécondité des Canadiennes de moins de 20 ans au XVIIe siècle. Mémoire de maîtrise, Université de Montréal (démographie), 1976, 218 p.

(2) Hubert Charbonneau, «Remariage et fécondité en Nouvelle-France», Mariage et remariage dans les populations du passé (Londres, Academic Press, 1981), 561-571.

(3) Hubert Charbonneau, Vie et mort de nos ancêtres (Montréal, Presses de l'Université de Montréal, 1975), 267 p.

(4) Louis Duchesne, Nuptialité et fécondabilité des veuves remariées: les Canadiennes au XVIIIe siècle. Mémoire de maîtrise, Université de Montréal, 1972, 88 p.

(5) Lorraine Gadoury, Yves Landry et Hubert Charbonneau, «Démographie différentielle en Nouvelle-France: villes et campagnes», $R H A F, 38,3$ (hiver 1985): 357-378.

(6) Jacques Henripin, La population canadienne au début du XVIIIe siècle, (Paris, Presses universitaires de France, 1954), 129 p.

(7) Yves Landry et Hubert Charbonneau, «Démographie différentielle et catégories sociales en Nouvelle-France», Actes du XVe Congrès international des sciences historiques (Bucarest, Editura Adademiei Republicii Socialisti Romania, 1982) 4: 1150-1163.

(8) Micheline Tremblay, La population de Beauport sous le régime français. Mémoire de mâ̂trise, Université de Montréal (démographie), 1981. 320 p.

(9) Lorraine Gadoury, «Le comportement démographique de la noblesse en Nouvelle-France: une piste de recherche», Cahiers d'histoire, 4,2 (1984): 5-28.

(10) Lorraine Bernier-Lemire, Neuville, des origines à 1762: étude démographique d'une paroisse rurale du Québec. Mémoire de maîtrise, Université de Montréal (démographie), 1982. 196 p.

(11) Serge Goudreau, La population de Cap-Santé, de Deschambault et de Grondines sous le régime français (1679-1762). Mémoire de maîtrise, Université de Montréal (démographie) 1984. $205 \mathrm{p}$. 
de $6,1 \%$ pour l'ensemble de la période. Ces données concordent avec celles des quelques chercheurs qui se sont arrêtés à mesurer le phénomène en Nouvelle-France (voir tableau 2): le pourcentage se situe autour de $4,5 \%$ au $17 \mathrm{e}$ (sauf pour les femmes de moins de vingt ans offrant un pourcentage légèrement inférieur) et il est plus élevé au $18 \mathrm{e}$ siècle.

Le phénomène n'offrait donc pas une ampleur considérable dans cette nouvelle colonie. Mais en cela, la Nouvelle-France ne différait guère de l'ancienne: J. Dupâquier ${ }^{18}$ estime à $6,7 \%$ en moyenne la fréquence des conceptions prénuptiales dans la France rurale de la période $1670-1739$.

\section{5 - L'évolution dans le temps et l'évolution de l'âge au mariage}

L'observation du phénomène dans le temps (voir tableau 3) nous fait constater une certaine stabilité avant 1690 puis une hausse par la suite. Indication d'un changement dans les moeurs intervenu au tournant du siècle? Non pas. Un seul facteur semble expliquer cette évolution: le marché matrimonial déséquilibré du $17 \mathrm{e}$ siècle. Cette situation de déséquilibre des sexes ${ }^{19}$, engendrée par l'immigration surtout masculine de cette colonie naissante, explique les fréquentations plutôt brèves des immigrantes (elles auront tout de même 5\% de conceptions prénuptiales au $17 \mathrm{e}$ siècle) et surtout les mariages précoces des Canadiennes de l'époque.

En ne retenant que les unions pour lesquelles nous connaissons l'âge exact des épouses (ce qui élimine en grande partie les immigrantes) nous constatons (voir graphique 1) que la croissance de la fréquence des conceptions prénuptiales est directement reliée à la hausse de l'âge au mariage des femmes. Les femmes jeunes ont une probabilité moins forte de concevoir avant leur mariage, à cause de leur moindre fécondité certes ${ }^{20}$, mais surtout, on peut le supposer, à cause du plus grand contrôle familial s'exerçant sur elles. Autrement dit: c'est parce qu'une grande partie des épouses du 17 e siècle étaient jeunes $(67,6 \%)$ de celles dont nous connaissons la date de naissance se sont mariées avant 20 ans) que les conceptions prénuptiales furent moins nombreuses à cette époque. En ne retenant que les femmes mariées à 20 ans et plus, la différence s'estompe entre les deux époques: 7,3\% de 1621 à 1699 , $7,5 \%$ de 1700 à 1724 .

18 Jacques Dupâquier, La population française aux XVIIe et XVIIIe siècles (Paris, Presses universitaires de France, 1979), 128 p.

19 Raymond Roy et Hubert Charbonneau, «La nuptialité en situation de déséquilibre de sexes: le Canada au XVIIe siècle», Annales de démographie historique (1978): 285-294.

${ }_{20}$ Hubert Charbonneau, «Les régimes de fécondité naturelle en Amérique du Nord: bilan et analyse des observations», Fécondité naturelle: niveaux et déterminants de la fécondité naturelle (Liège, Ordina Editions, 1979), 441-491. 
TABLEAU 3

Conceptions prénuptiales par périodes

Canada 1621-1724

\begin{tabular}{|c|c|c|c|c|c|c|c|c|c|}
\hline & \multicolumn{9}{|c|}{ Périodes } \\
\hline & $\begin{array}{r}\text { avant } \\
1660\end{array}$ & $\begin{array}{l}1660- \\
1669\end{array}$ & $\begin{array}{l}1670- \\
1679\end{array}$ & $\begin{array}{l}1680- \\
1689\end{array}$ & $\begin{array}{l}1690- \\
1699\end{array}$ & $\begin{array}{l}1700- \\
1709\end{array}$ & $\begin{array}{l}1710- \\
1719\end{array}$ & $\begin{array}{l}1720- \\
1724\end{array}$ & $\begin{array}{l}1621- \\
1724\end{array}$ \\
\hline Nombre de premières naissances & 240 & 434 & 509 & 740 & 1131 & 1243 & 1779 & 1107 & 7183 \\
\hline $\begin{array}{l}\text { Intervalles protogénésiques } \\
\text { inférieurs à huit mois révolus }\end{array}$ & 10 & 12 & 22 & 32 & 64 & 104 & 118 & 74 & 736 \\
\hline $\begin{array}{l}\text { Proportion de conceptions } \\
\text { prénuptiales }(\%)\end{array}$ & 4,2 & 2,8 & 4,3 & 4,3 & 5,7 & 8,4 & 6,6 & 6,7 & 6,1 \\
\hline
\end{tabular}




\section{GRAPHIQUE 1}

Évolution de la fréquence des conceptions prénuptiales et de l'âge au mariage des épouses -

Canada 1621-1724

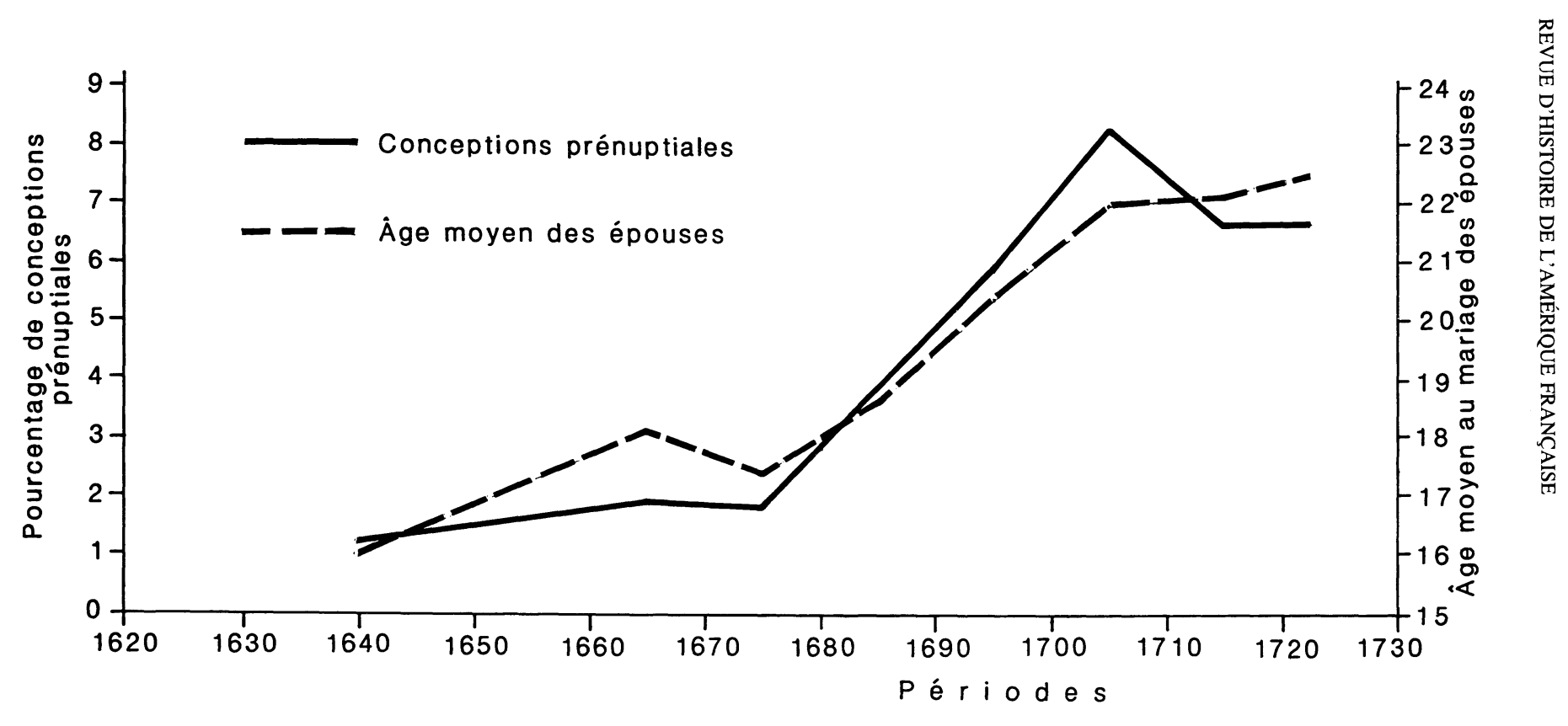


TABLEAU 4

Conceptions prénuptiales selon l'état matrimonial de l'épouse

et selon son âge au mariage - Canada 1621-1724

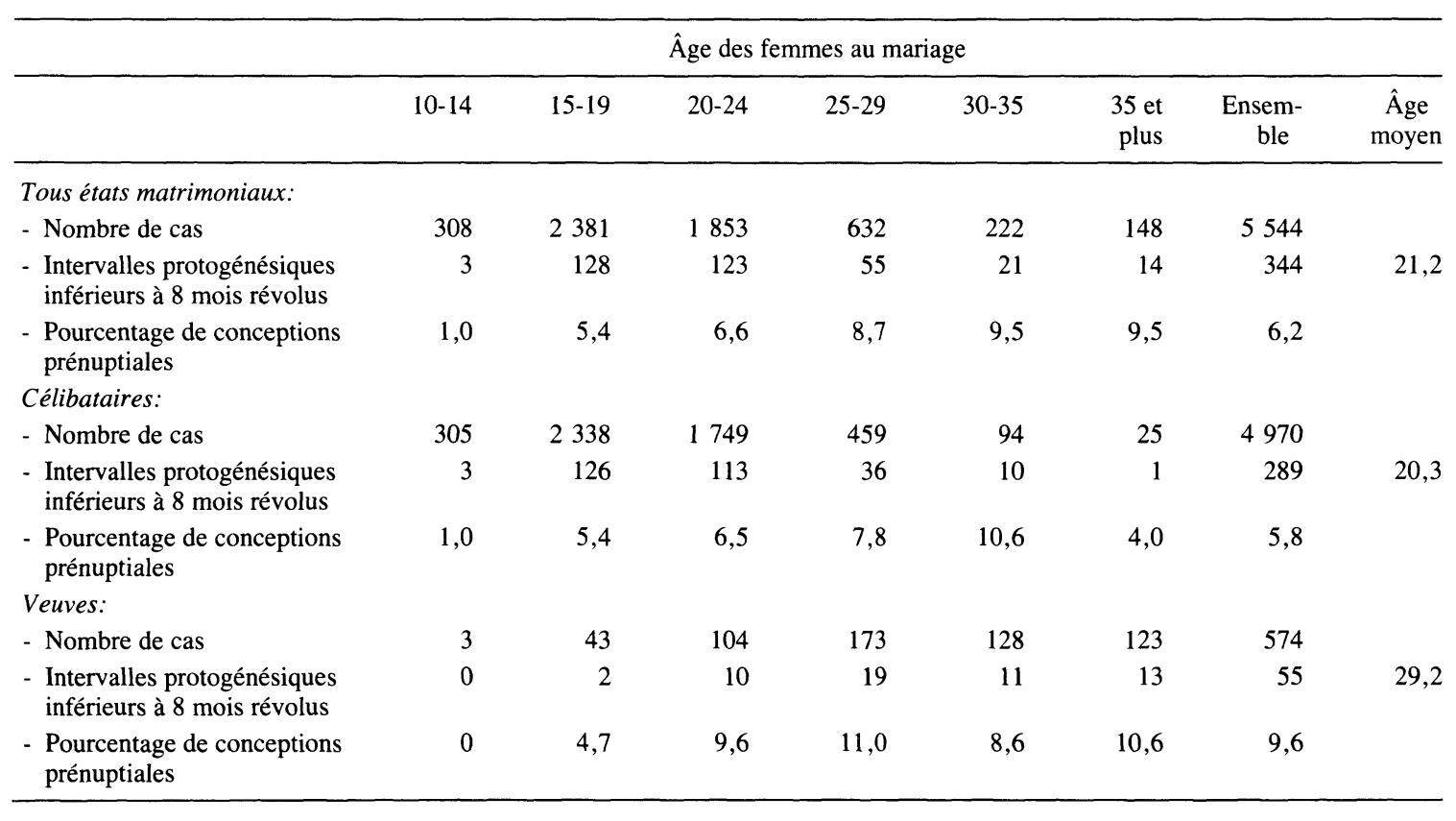


Ces veuves, tout au moins celles épousant des célibataires (voir tableau 5), ont d'ailleurs un comportement plus libre que les célibataires. L'âge joue sûrement un rôle dans la différence selon les états matrimoniaux, les veuves étant plus âgées, mais n'explique pas entièrement cette différence: entre 20 et 30 ans une veuve connaissait un plus grand risque de concevoir avant le mariage qu'une célibataire du même âge (tableau 4), mais à 30 ans et plus le comportement des célibataires est analogue à celui des veuves.

TABLEAU 5

Conceptions prénuptiales selon l'état matrimonial combiné des conjoints

Canada 1621-1724

\begin{tabular}{lllccc}
\hline $\begin{array}{c}\text { Époque du } \\
\text { mariage }\end{array}$ & \multicolumn{1}{c}{$\begin{array}{c}\text { État matrimonial } \\
\text { Épouse }\end{array}$} & Époux & $\begin{array}{c}\text { Nombre de } \\
\text { premières } \\
\text { naissances }\end{array}$ & $\begin{array}{c}\text { Intervalles } \\
\text { protogénésiques } \\
\text { inférieurs à } \\
8 \text { mois révolus }\end{array}$ & $\begin{array}{c}\% \text { de } \\
\text { conceptions } \\
\text { prénuptiales }\end{array}$ \\
\hline \multirow{2}{*}{$1621-1699$} & célibataire & célibataire & 2485 & 94 & 3,8 \\
& célibataire & veuf & 168 & 6 & 3,6 \\
& veuve & célibataire & 343 & 36 & 10,5 \\
& veuve & veuf & 58 & 4 & 6,9 \\
\hline \multirow{2}{*}{$1700-1724$} & célibataire & célibataire & 3238 & 234 & 7,2 \\
& célibataire & veuf & 452 & 19 & 4,2 \\
& veuve & célibataire & 333 & 39 & 11,7 \\
& veuve & veuf & 106 & 4 & 3,8 \\
\hline \multirow{2}{*}{$1621-1724$} & célibataire & célibataire & 5723 & 328 & 5,7 \\
& célibataire & veuf & 620 & 25 & 4,0 \\
& veuve & célibataire & 676 & 75 & 4,1 \\
& veuve & veuf & 164 & 8 & 6,1 \\
\hline
\end{tabular}

Ces constatations selon l'âge et l'état matrimonial antérieur des épouses ne sont pas nouvelles. Tant pour la Nouvelle-France ${ }^{21}$ que pour l'ancienne ${ }^{22}$, de telles observations ont été faites. Ces différences de comportement s'expliqueraient aisément. Dans une société interdisant les rapports sexuels hors mariage, c'est surtout l'ampleur du contrôle social sur les individus qui peut expliquer le respect d'une telle norme. Or les filles jeunes et célibataires étaient sans aucun doute plus fermement sous le joug familial que les filles plus âgées ou les veuves. L'observation en fonction de la survie des parents au mariage permet d'asseoir plus solidement une telle explication.

\footnotetext{
21 Charbonneau, Vie et mort...

22 Hubert Charbonneau, Tourouvre-au-Perche aux XVIIe et XVIIIe siècles (Paris, Presses universitaires de France, 1970), 423 p.
} 
TABLEAU 6

Conceptions prénuptiales selon l'état matrimonial de l'épouse et la survie de son père - Canada 1621-1724

\begin{tabular}{|c|c|c|}
\hline & $\begin{array}{c}\text { Père } \\
\text { vivant }\end{array}$ & $\begin{array}{l}\text { Père } \\
\text { mort }\end{array}$ \\
\hline \multicolumn{3}{|l|}{ Célibataires } \\
\hline $\begin{array}{l}\text { Nombre de premières } \\
\text { naissances }\end{array}$ & 2830 & 1780 \\
\hline $\begin{array}{l}\text { Intervalles protogénésiques } \\
\text { inférieurs à } 8 \text { mois révolus }\end{array}$ & 143 & 124 \\
\hline$\%$ de conceptions prénuptiales & 5,1 & 7,0 \\
\hline \multicolumn{3}{|l|}{ Veuves } \\
\hline $\begin{array}{l}\text { nombre de premières } \\
\text { naissances }\end{array}$ & 217 & 304 \\
\hline $\begin{array}{l}\text { Intervalles protogénésiques } \\
\text { inférieurs à } 8 \text { mois révolus }\end{array}$ & 17 & 34 \\
\hline$\%$ de conceptions prénuptiales & 7,8 & 11,2 \\
\hline
\end{tabular}

Chez les célibataires, et encore plus chez les veuves, la fréquence des conceptions prénuptiales est plus élevée lorsque le père est mort (voir tableau 6). L'âge peut certes ici jouer un rôle. Nous pouvons isoler ce facteur, tout au moins pour les célibataires dont le nombre permet des subdivisions selon le groupe d'âges (voir tableau 7). Peu importe

\section{TABLEAU 7}

Conceptions prénuptiales selon la survie des pères pour les épouses célibataires nées au Canada - Canada 1621-1724

\begin{tabular}{|c|c|c|c|c|c|c|}
\hline \multirow[b]{2}{*}{$\begin{array}{l}\text { Survie des parents } \\
\text { de l'épouse }\end{array}$} & \multirow[b]{2}{*}{$10-14$} & \multicolumn{5}{|c|}{ Âge au mariage des épouses } \\
\hline & & $15-19$ & $20-24$ & $25-29$ & $\begin{array}{l}30 \text { et } \\
\text { plus }\end{array}$ & $\begin{array}{c}\text { Ensem- } \\
\text { ble }\end{array}$ \\
\hline \multicolumn{7}{|l|}{ Père vivant: } \\
\hline $\begin{array}{l}\text { - Nombre de premières } \\
\text { naissances }\end{array}$ & 206 & 1433 & 974 & 186 & 31 & 2830 \\
\hline $\begin{array}{l}\text { - Intervalles protogé- } \\
\text { nésiques inférieurs } \\
\text { à } 8 \text { mois révolus }\end{array}$ & 2 & 72 & 53 & 14 & 2 & 143 \\
\hline $\begin{array}{l}\text { - Pourcentage de con- } \\
\text { ceptions prénuptiales }\end{array}$ & 1,0 & 5,0 & 5,4 & 7,5 & 6,5 & 5,1 \\
\hline \multicolumn{7}{|l|}{ Père mort: } \\
\hline $\begin{array}{l}\text { - Nombre de premières } \\
\text { naissances }\end{array}$ & 73 & 740 & 660 & 229 & 78 & 1780 \\
\hline $\begin{array}{l}\text { - Intervalles protogé- } \\
\text { nésiques inférieurs } \\
\text { à } 8 \text { mois révolus }\end{array}$ & 1 & 47 & 48 & 19 & 9 & 124 \\
\hline $\begin{array}{l}\text { - Pourcentage de con- } \\
\text { ceptions prénuptiales }\end{array}$ & 1,4 & 6,4 & 7,3 & 8,3 & 11,5 & 7,0 \\
\hline
\end{tabular}


l'âge, les conceptions prénuptiales sont plus fréquentes lorsque le père est décédé au moment du mariage, ce qui confirme notre hypothèse.

\section{7 - Immigrantes et Canadiennes}

Si les Françaises (voir tableau 8), arrivées et mariées en quasitotalité avant 1700 , ont une moins forte propension à concevoir avant le mariage que les Canadiennes c'est sûrement parce qu'elles sont arrivées et se sont mariées à l'époque du déséquilibre des sexes (85\% d'entre elles se sont mariées avant 1681) qui les obligea à convoler rapidement, limitant donc les fréquentations porteuses de tentations. Elles ont tout de même $5 \%$ de conceptions prénuptiales, ce qui laisse croire que les fréquentations ne furent pas toujours si brèves pour ces immigrantes dont $20 \%$ étaient veuves.

TABLEAU 8

Conceptions prénuptiales selon le pays de naissance des épouses

Canada 1621-1724

\begin{tabular}{llccc}
\hline $\begin{array}{l}\text { Période du } \\
\text { mariage }\end{array}$ & $\begin{array}{l}\text { Pays de } \\
\text { naissance }\end{array}$ & $\begin{array}{c}\text { Nombre de } \\
\text { premières } \\
\text { naissances }\end{array}$ & $\begin{array}{c}\text { Intervalles protogé- } \\
\text { nésiques inférieurs } \\
\text { à } 8 \text { mois révolus }\end{array}$ & $\begin{array}{c}\text { Pourcentage de } \\
\text { conceptions } \\
\text { prénuptiales }\end{array}$ \\
\hline $1621-1699$ & France & 953 & 48 & 5,0 \\
& Canada & 2082 & 90 & 4,3 \\
$1700-1724$ & France & 58 & 1 & 1,7 \\
& Canada & 4044 & 288 & 7,1 \\
\hline $1621-1724$ & France & 1011 & 49 & 4,8 \\
& Canada & 6126 & 378 & 6,2 \\
\hline
\end{tabular}

Quant aux Canadiennes, nous avons déjà observé que leur âge au mariage explique la plus faible proportion de conceptions prénuptiales du 17 e siècle. Ce n'est qu'au 18e siècle que leur comportement se démarque de celui des immigrantes.

\section{8 - Géographie des conceptions prénuptiales}

\section{A - Les conceptions prénuptiales dans les trois gouvernements}

Le comportement des couples n'était pas homogène dans toute la vallée du Saint-Laurent, tout au moins après 1700 où une nette distinction s'établit entre d'une part le gouvernement de Québec et d'autre part les gouvernements de Trois-Rivières et Montréal où les conceptions prénuptiales étaient plus nombreuses (voir tableau 9). Ni l'âge moyen des épouses, ni la proportion de veuves impliquées dans ces mariages n'expliquent ces différences. Peut-être que la mouvance des voyageurs engagés dans la traite des fourrures créait un climat plus 
propice aux conceptions hors mariage, tout au moins à Montréal, région frontalière dont Lahontan écrivait au 17e siècle: «Vous seriez surpris de voir les débauches, les festins, les jeux et les dépenses que ces coureurs de bois font tant en habits qu'en femmes, dès qu'ils sont arrivés. ${ }^{23}$

Mais ce climat ne se remarquerait, du point de vue des conceptions prénuptiales, qu'après 1700 .

TABLEAU 9

Conceptions prénuptiales selon les trois gouvernements, avant et après 1700 - Canada 1621-1724

\begin{tabular}{llccc}
\hline Période & Gouvernement & $\begin{array}{c}\text { Nombre de } \\
\text { premières } \\
\text { naissances }\end{array}$ & $\begin{array}{c}\text { Intervalles protégé- } \\
\text { nésiques inférieurs } \\
\text { à 8 mois révolus }\end{array}$ & $\begin{array}{c}\% \text { de } \\
\text { conceptions } \\
\text { prénuptiales }\end{array}$ \\
\hline $1621-$ & Québec & 1969 & 92 & 4,7 \\
1699 & Trois- & 218 & 9 & 4,1 \\
& Rivières & & 39 & 4,5 \\
$1700-$ & Montréal & 867 & 131 & 5,9 \\
1724 & Québec & 2228 & 32 & 9,4 \\
& Trois- & 340 & 133 & 8,5 \\
& Rivières & & 223 & 5,3 \\
$1621-$ & Montréal & 1561 & 41 & 7,4 \\
1724 & Québec & 4197 & 172 & 7,1 \\
& Trois- & 558 & 436 & 6,1 \\
\hline
\end{tabular}

\section{$B$ - Villes et campagnes}

Nous avons assimilé les paroisses Notre-Dame de Québec, NotreDame de Montréal et l'Immaculée-Conception des Trois-Rivières à des paroisses urbaines. Certes ce n'est pas parfaitement exact. Mais si nombre de ruraux habitaient ces paroisses, celles-ci, par leurs fonctions administratives, sociales (santé, éducation) et économiques, peuvent être considérées plus comme des villes que comme des campagnes.

Nous observons $7,3 \%$ de conceptions avant mariage en ville contre $5,3 \%$ en milieu rural (voir tableau 10). Mais la différence ne semble vraie qu'après 1700 où près de deux fois plus d'épouses se marient enceintes dans les villes. Les données pour le $17 \mathrm{e}$ siècle peuvent être biaisées: nous avons assimilé le lieu d'enregistrement du mariage au lieu de résidence des conjoints; or cette assimilation n'est certes pas

23 Louis-Armand de Lom d'Arce, baron de Lahontan, Nouveaux voyages en Amérique septentrionale (Montréal, Hexagone/Minerve, 1983), 83. 
TABLEAU 10

Conceptions prénuptiales selon le milieu urbain/rural avant et après 1700 - Canada 1621-1724

\begin{tabular}{|c|c|c|c|c|c|c|c|}
\hline Période & & $\begin{array}{c}\text { (1) } \\
\text { Québec }\end{array}$ & $\begin{array}{c}(2) \\
\text { Trois- } \\
\text { Rivières }\end{array}$ & $\begin{array}{c}\text { (3) } \\
\text { Montréal }\end{array}$ & $\begin{array}{c}(4)=(1+2+3) \\
\text { Villes }\end{array}$ & $\begin{array}{c}(5) \\
\text { Campagnes }\end{array}$ & $\begin{array}{c}(6)=(4+5) \\
\text { Ensemble }\end{array}$ \\
\hline \multirow[t]{4}{*}{$1621-1699$} & Nombre de cas & 1020 & 88 & 525 & 1633 & 1421 & 3054 \\
\hline & $\begin{array}{l}\text { Intervalles protogénésiques } \\
\text { inférieurs à } 8 \text { mois révolus }\end{array}$ & 52 & 7 & 18 & 77 & 63 & 140 \\
\hline & $\begin{array}{l}\text { Pourcentage de conceptions } \\
\text { prénuptiales }\end{array}$ & 5,1 & 8,0 & 3,4 & 4,7 & 4,4 & 4,6 \\
\hline & Pourcentage de veuves & 15 & 17 & 14 & 15 & 12 & 13 \\
\hline \multirow[t]{4}{*}{$1700-1724$} & Nombre de cas & 635 & 71 & 585 & 1291 & 2838 & 4129 \\
\hline & $\begin{array}{l}\text { Intervalles protogénésiques } \\
\text { inférieurs à } 8 \text { mois révolus }\end{array}$ & 60 & 15 & 60 & 135 & 161 & 296 \\
\hline & $\begin{array}{l}\text { Pourcentage de conceptions } \\
\text { prénuptiales }\end{array}$ & 9,4 & 21,1 & 10,3 & 10,5 & 5,7 & 7,2 \\
\hline & Pourcentage de veuves & 13 & 7 & 11 & 12 & 10 & 11 \\
\hline \multirow[t]{4}{*}{$1621-1724$} & Nombre de cas & 1655 & 159 & 1110 & 2924 & 4259 & 7183 \\
\hline & $\begin{array}{l}\text { Intervalles protogénésiques } \\
\text { inférieurs à } 8 \text { mois révolus }\end{array}$ & 112 & 22 & 78 & 212 & 224 & 436 \\
\hline & $\begin{array}{l}\text { Pourcentage de conceptions } \\
\text { prénuptiales }\end{array}$ & 6,8 & 13,8 & 7,0 & 7,3 & 5,3 & 6,1 \\
\hline & Pourcentage de veuves & 14 & 13 & 12 & 13 & 11 & 12 \\
\hline
\end{tabular}


exacte, particulièrement pour le $17 \mathrm{e}$ siècle où nombre de paroisses rurales n'étaient pas encore érigées et où, par conséquent, des ruraux venaient se marier dans les paroisses urbaines. Lorraine Gadoury, Yves Landry et Hubert Charbonneau ${ }^{24}$ ont en effet trouvé, pour leur échantillon du $17 \mathrm{e}$ siècle, un peu plus de conceptions prénuptiales chez les couples urbains $(4,8 \%)$ que chez les ruraux $(3,4 \%)$. Ni l'âge moyen des épouses ni leur état matrimonial antérieur n'expliquent ces différences entre villes et campagnes. Quant aux villes, les différences entre elles ne sont pas significatives.

\section{9 - Les différences sociales}

Toute une étude sur le comportement différentiel en matière de conceptions prénuptiales selon les groupes sociaux reste à faire. A cause des lacunes considérables dans les mentions de professions pour les époux et les pères des conjoints, à cause également de la présence d'éléments perturbateurs qui nuisent à l'interprétation des différences, une telle analyse différentielle selon le groupe social devient presque impossible. Ainsi, par exemple, pour les époux agriculteurs, $96 \%$ des unions connues datent du $17 \mathrm{e}$ siècle: leur faible pourcentage de conceptions prénuptiales $(25 / 762=3,3 \%)$ peut alors s'expliquer par ce fait et non par un comportement plus «sage» des membres de cette catégorie. On peut cependant noter une moins forte propension à concevoir avant le mariage chez les filles de notables $(24 / 570=4,2 \%)$ - mais non chez les fils de ces mêmes notables $(38 / 491=7,7 \%)$ - que chez les filles des autres groupes $(193 / 3102=6,2 \%)$.

Une catégorie d'unions se détache cependant nettement de l'ensemble: celle où le conjoint est militaire; la situation particulière des militaires qui devaient avoir la permission de leurs supérieurs pour se marier explique sûrement la forte proportion des conceptions prénuptiales dans leurs unions (10,2\% pour l'ensemble de la période, et $20,2 \%$ pour la seule décennie 1700-1709).

\section{0 - La saison des amours}

Jacques Henripin ${ }^{25}$ avait montré que le mouvement saisonnier des premières conceptions (premières naissances moins neuf mois) dépendait du mouvement saisonnier des mariages. Mais parmi ces premières conceptions, certaines ne peuvent évidemment être influencées de la même façon par le moment du mariage puisqu'elles le précèdent. Au graphique 2 nous pouvons observer que le mouvement saisonnier des conceptions prénuptiales diffère nettement de celui des conceptions

24 Lorraine Gadoury, Yves Landry et Hubert Charbonneau, «Démographie différentielle en Nouvelle-France: villes et campagnes», Revue d'histoire de l'Amérique française, 38,3 (hiver 1985): 357-378.

25 Henripin, op cit. 
GRAPHIQUE 2

Mouvement mensuel des conceptions donnant lieu à une naissance de rang 1

Canada 1621-1724

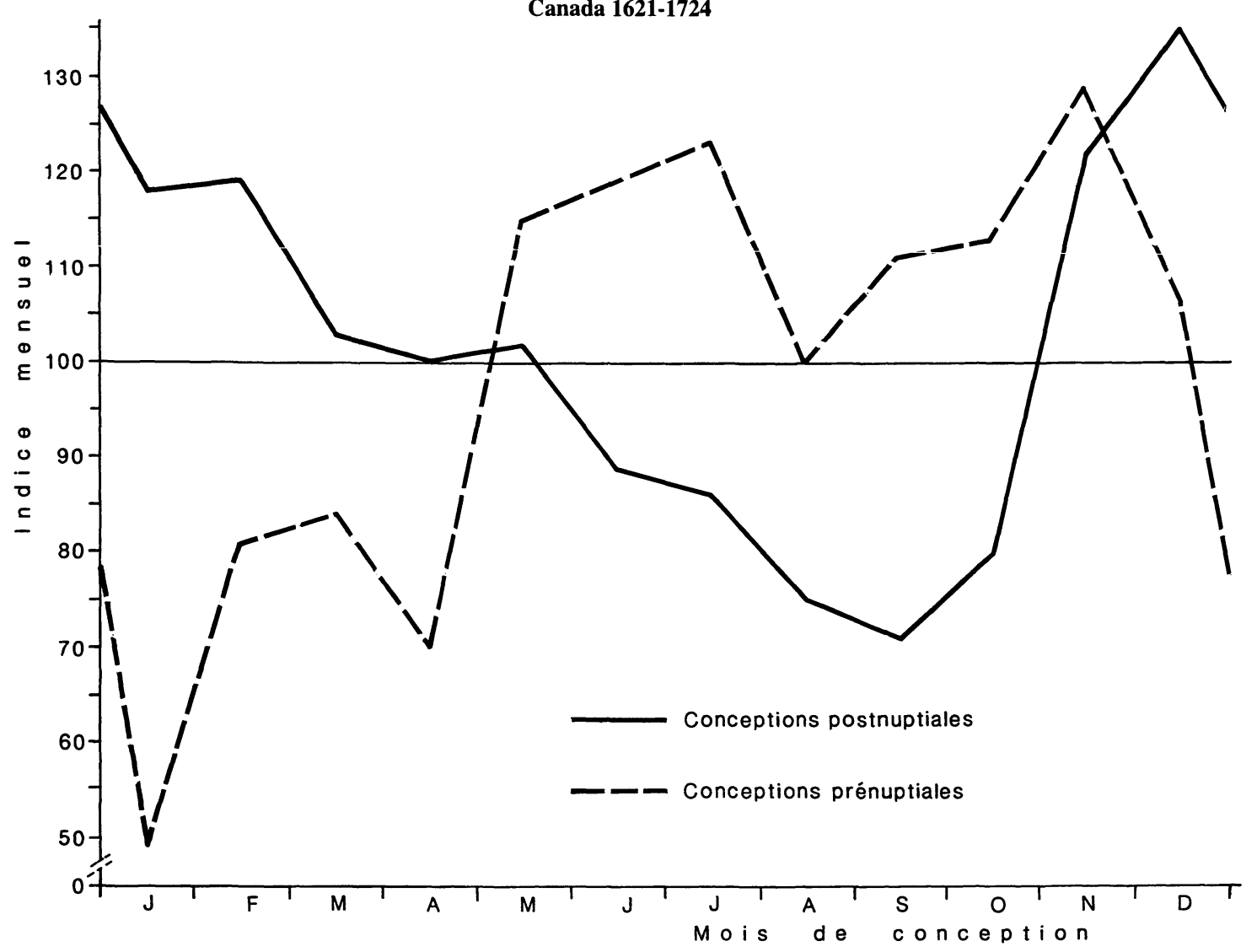

న

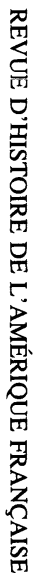


postnuptiales: les conceptions prénuptiales ont lieu aux temps chauds (mai, juin, juillet) et en automne (septembre, octobre, novembre) cependant que les autres sont des conceptions d'hiver, suivant en cela le mouvement saisonnier des mariages (on se mariait surtout en novembre, janvier et février).

\section{1 - Mariages provoqués, mariages anticipés}

L'existence des conceptions prénuptiales témoigne certes, pour les couples concernés, d'un non-respect des normes religieuses concernant la sexualité hors mariage. Cela signifie-t-il nécessairement qu'un grand nombre de fiancés se permettait des privautés avant les noces? On sait que dans certaines régions européennes des traditions anciennes laissaient aux jeunes gens une plus ou moins grande liberté de fréquentation (visites nocturnes dans les pays scandinaves et germaniques, cohabitation des fiancés en Corse ou en Angleterre). Avant les prescriptions du Concile de Trente, qui ne s'imposèrent sûrement pas tout de suite partout, les fiançailles étaient vécues comme le vrai début de l'union charnelle du couple ${ }^{26}$.

Une telle tradition exista-t-elle en Nouvelle-France? On peut difficilement penser en tout cas qu'elle était généralisée (le pourcentage de conceptions prénuptiales serait plus élevé) mais le jugement de Mgr de Saint-Vallier témoignerait de certaines libertés prises par les fiancés:

L'on ne doit jamais permettre aux fiancés de demeurer dans une même maison, ni de se fréquenter familièrement. La différence que nous avons remarquée être dans plusieurs Paroisses de ce Diocèse, dans le plus grand nombre desquelles on n'observoit point la solennité des Fiançailles, et dans les autres on l'observoit, et les abus que nous y avons remarqués, nous ont fait prendre la résolution de les retrancher. ${ }^{27}$

Ces fiançailles officielles, faites devant curé, furent en effet interdites en 1698 et le Rituel de 1703 ne les accepta qu'à la condition qu'elles ne donnent pas lieu à des abus et qu'elles soient célébrés «avec modestie» ${ }^{28}$.

On connaît aussi cette tradition du mariage «à la gaumine» importée de France. Cela consistait pour un couple à se présenter à l'église avec deux témoins et à se consacrer mari et femme: comme il y avait présence du prêtre, les conjoints se considéraient comme légitimement mariés. Mais ni l'Église ni le gouvernement civil n'acceptèrent ces mariages qui, à partir de 1717, amenèrent l'excommunication de ceux

\footnotetext{
26 Jean-Louis Flandrin, Les amours paysannes (Paris, Gallimard/Julliard, 1975), 256 p.

27 Saint-Vallier, Rituel..., 289.

28 Paul-André Leclerc, «Le mariage sous le Régime français», Revue d'histoire de l'Amérique française, 13,2 (septembre 1959): 230-246; 13,3 (décembre 1959): 374-401; 13,4 (mars 1960): $525-543$ et 14,1 (juin 1960): 34-60; 14,2 (septembre 1960): 226-245.
} 
qui le pratiquaient ${ }^{29}$. Ces mariages furent-ils nombreux? On peut penser, sans doute, que ces mariages demeurèrent un épiphénomène marginal; et comme ils étaient validés quelque temps plus tard, la morale de l'époque était sauve. Mais dans de tels cas les épouses risquaient de devenir enceintes avant ce second mariage, le seul officiel: quelquesunes des conceptions prénuptiales de l'époque seraient probablement reliées à ce type d'unions.

Quant aux fréquentations, il semble bien qu'elles duraient rarement longtemps, si l'on se fie au témoignage de Lahontan:

Or ce qui fait qu'on se marie facilement en ce Pais-là c'est la difficulté de pouvoir converser avec les personnes de l'autre sexe. Il faut se déclarer aux Pères et Mères au bout de quatre visites qu'on fait à leurs filles; il faut parler de mariage ou cesser tout commerce, sinon la médisance attaque les uns et les autres comme il faut. ${ }^{30}$

Les historiens reprennent ce fait, et ils ne manquent pas de revenir sur l'arrivée des Filles du Roy et de l'obligation faite par Talon aux célibataires de se marier dans les quinze jours après l'arrivée des vaisseaux.

On peut cependant se demander s'il en fut toujours ainsi. Au début de la colonie, à l'époque du déséquilibre des sexes et de l'arrivée des immigrants, on conçoit aisément que les fréquentations aient été brèves. Mais par la suite, quand filles et garçons nés dans une paroisse se connaissaient depuis leur enfance, tel était-il le cas? L'évolution à la hausse de l'âge au mariage des filles témoignerait d'une plus longue période de fréquentation. Chose certaine, les fréquentations brèves, et le contrôle social qu'elles impliquaient ${ }^{31}$, rendaient difficiles les conceptions d'avant le mariage.

Mais les conceptions prénuptiales peuvent témoigner d'autre chose que de la liberté de comportement des fiancés: certaines conceptions pouvaient en effet obliger certains couples à régulariser, en se mariant, une situation mal vue socialement. Ne connaissant pas l'histoire particulière des couples concernés, peut-on tenter d'établir statistiquement une distinction entre ces deux catégories d'unions à conceptions prénuptiales?

Les naissances des quatre premiers mois correspondent à des conceptions intervenues 6 à 9 mois avant le mariage: dans ces cas il ne fait pas de doute que les femmes ont amplement eu le temps de connaitre leur état et il nous semble permis de supposer que les mariages ont été

\footnotetext{
29 Ibid.

30 Cité par R.-L. Séguin, op. cit., 359.

31 «Les fréquentations entre jeunes gens de différents sexes échappent difficilement à l'oeil inquisiteur du chaperon», R.-L. Séguin, op. cit., 365.
} 
provoqués par ces grossesses. Les conceptions intervenues dans les trois mois précédant le mariage n'ont sûrement pas provoqué celui-ci (compte tenu du délai nécessaire pour prendre conscience de l'état de grossesse, de décider le conjoint et/ou les parents, etc.). Il n'est pas trop risqué de juger ces conceptions comme des libertés que se sont permises des «promis». Quant aux naissances des mois 4 et 5 (conceptions quatre à cinq mois avant le mariage), elles peuvent avoir provoqué le mariage ou avoir anticipé sur celui-ci: comment distinguer? Nous avons choisi d'assimiler les naissances du mois 4 révolu à celles du premier groupe (mariages provoqués) et celles du mois 5 au deuxième groupe (mariages anticipés). Cette délimitation n'est certes pas parfaite mais permet tout de même d'estimer, au moins approximativement, la proportion de mariages provoqués. On constate alors que $60 \%$ des conceptions prénuptiales correspondent au second type (mariages anticipés): on pourrait en conclure, avec Jean-Pierre Bardet ${ }^{32}$, que «la relative liberté sexuelle» de nos ancêtres «s'inscrivait en fait dans une perspective nuptiale».

Notons cependant que pour les unions impliquant un conjoint militaire la proportion s'inverse: $60 \%$ des conceptions prénuptiales de ces unions sont intervenues 5 à 9 mois avant le mariage, ce qui concorde avec l'observation déjà faite sur la permission que devaient avoir les militaires pour se marier, situation qui impliquait certainement un délai.

\section{2 - Conceptions prénuptiales et naissances illégitimes}

Par certaines caractéristiques le phénomène des conceptions prénuptiales rejoint celui des naissances illégitimes ${ }^{33}$. Ainsi, dans les deux cas, la fréquence est plus élevée au $18 \mathrm{e}$ siècle qu'au $17 \mathrm{e}(0,83 \%$ de naissances illégitimes avant $1700,1,49 \%$ entre 1700 et 1729). Dans les deux cas également la fréquence croît en même temps que l'âge moyen des épouses et la décennie 1700-1709 se démarque par son pourcentage plus élevé de naissances illégitimes $(1,74 \%)$ et de conceptions prénuptiales $(8,4 \%)$. L'illégitimité, tout comme la prénuptialité, est plus un phénomène urbain que rural et le mouvement saisonnier des naissances illégitimes se rapproche de celui des naissances issues de conceptions prénuptiales (conceptions surtout des temps chauds: printemps et été).

On sait que la fréquence des naissances illégitimes se calcule en rapportant celles-ci à l'ensemble des naissances, quel que soit le rang de ces dernières. Mais comme le fait remarquer fort justement l'historien Flandrin ${ }^{34}$, c'est aux seules premières naissances qu'il faudrait rapporter les naissances illégitimes, puisqu'elles sont surtout des nais-

32 Jean-Pierre Bardet, Rouen aux XVIIe et XVIIIe siècles. Les mutations d' un espace social (Paris, SEDES, 1983), 2 volumes, 421 et $197 \mathrm{p}$.

34 Paquette, op.cit.

34 Flandrin, op . cit. 
sances de rang 1. Inspiré par cette idée, nous avons estimé une proportion de premières conceptions illégitimes, que celles-ci aboutissent à des naissances hors mariage (naissances illégitimes) ou à l'intérieur du cadre conjugal (conceptions prénuptiales).

Pour ce faire nous avons utilisé les données de L. Paquette ${ }^{35}$. En rapportant les naissances illégitimes de rang 1 des filles qui se sont par la suite mariées à l'ensemble des mariages féconds de la période circonscrite par L. Paquette (1621-1729) et en faisant l'hypothèse que la proportion de conceptions prénuptiales ne s'élève pas entre 1725 et 1729 , nous avons obtenu une proportion de $11,4 \%$ de femmes ayant conçu avant leur mariage. En gros, la moitié de ces conceptions illégitimes ont abouti à des naissances illégitimes et l'autre moitié à des conceptions prénuptiales.

\section{CONCLUSION}

Les données, tant en ce qui concerne les naissances illégitimes que les conceptions prénuptiales, nous permettent de mieux connaître les moeurs de nos ancêtres en matière sexuelle. Que pouvons-nous en conclure?

Si l'on peut constater, par le biais des conceptions prénuptiales et des naissances illégitimes, une relative importance des rapports sexuels (féconds) hors mariage dans cette Nouvelle-France d'avant 1730, il nous faut bien admettre que plus de $90 \%$ (si on ne tient compte que des conceptions prénuptiales) ou près de $90 \%$ (si on tient compte également des naissances illégitimes) des épouses ont été fécondes uniquement à l'intérieur du cadre conjugal. Ce qui nous apparaît représenter un assez large consensus social vis-à-vis du neuvième commandement. Certes, naissances illégitimes et conceptions prénuptiales ne nous permettent de connaître que les rapports sexuels hors mariage féconds. Il resterait à estimer la proportion de rapports sexuels inféconds. De même que certains aspects des moeurs sexuelles de l'époque (tels que la bigamie, la prostitution, l'adultère, etc.) restent à connaître. Mais tout ceci exigerait d'autres études. 